\title{
Analysis and Research of Dynamic Observation Transformation Based on the Size of 3D Scene
}

\author{
Wei Wang ${ }^{1,2}$ and Jianxun Chen ${ }^{1,2}$ \\ 1. College of Computer Science and Technology, Wuhan University of Science and Technology. \\ 2. Hubei Province Key Laboratory of Intelligent Information Processing and Real-time Industrial \\ System, Wuhan, CHINA, 430065 \\ wei.wang.wust@qq.com
}

\begin{abstract}
Key words: Observation transform; View; View position; Observation volume; Observation matrix; Projection matrix
\end{abstract}

\begin{abstract}
In the actual project, when the three-dimensional scene is observed, the three-dimensional scene and the observer appear in the different observation matrix (viewpoint position, projection center and its upward direction), because the size of the observed object is not completely surrounded. Dislocation relationship, resulting in the view display is missing, not the ideal projection view, to the development of the system to bring confusion and interference. Therefore put forward a feasible method: in the first place, according to the actual size of $3 \mathrm{~d}$ scene, define a completely view sphere to surround the scene, spherical reference point as the point of view, its center point as center of view, this two points decided the projection direction; Finally on the projection direction, according to the $3 \mathrm{~d}$ scene actual size y determining the minimum observation volume to surround the $3 \mathrm{~d}$ scene. Experimental results show that the transformation method for $3 \mathrm{~d}$ scene observation, can easily determine the view matrix and projection matrix, improve the efficiency of the development of $3 \mathrm{~d}$ graphics system.
\end{abstract}

\section{Introduction}

After building the graphic objects in the virtual world, can by observing the transformation process, generate virtual images of different viewpoints. In modern computer graphics, defined from $3 \mathrm{~d}$ virtual world scene geometric configuration of the $2 \mathrm{~d}$ image map process called view [1].

The view of modern computer graphics system is very complex, may need a lot of parameters, including the definition of view matrix and projection matrix. Generally speaking, the view point of view in the virtual world, from a particular direction and orientation of observation in the virtual world; Each view has some custom properties, such as position location, direction, view center, horizon, front and back end cutting plane, and the size of the projection plane, etc. (Refer with: Fig. 1). Literature $[4,5]$ for a variety of observation location observation transformation puts forward the related research, literature [6,7] to observe the changing made detailed analysis; Literature [8] proposed creating a viewpoint ball, through choosing dynamically observe the changing point of view to achieve.

In practice, at a particular point of view, observation under the direction and orientation, the projection matrix defined by $2 \mathrm{~d}$ image is not comprehensive, affect the observation effect. So, here we discuss under the specified observation matrix, to determine the best projection matrix, able to complete the three dimensional scene is mapped to two dimensional image, improve the development efficiency of $3 \mathrm{~d}$ graphics. 


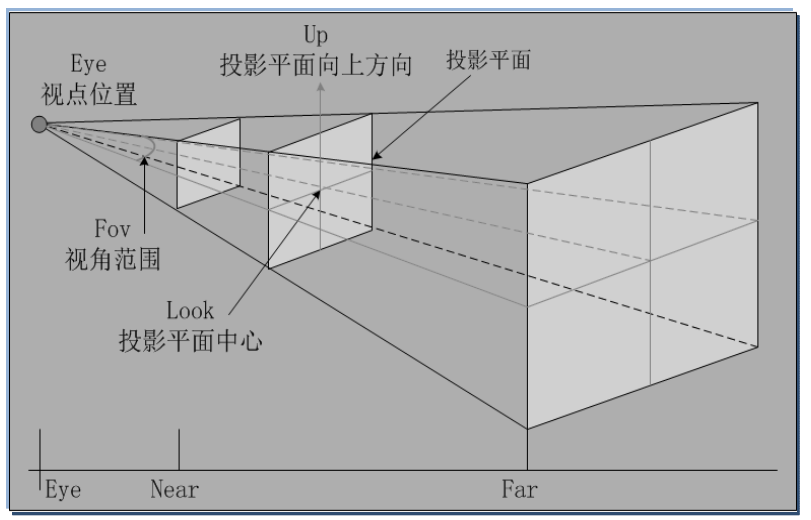

Figure 1.View model

\section{Technical Principle}

Eventually get the $3 \mathrm{~d}$ scene two-dimensional map image; firstly it needs to build $3 \mathrm{~d}$ scene according to the coordinate system, and mapping a part of the scene in the world coordinate system to the device coordinate system by view transformation. Observe transformation which needs the world coordinate system and observe the coordinate transformation and the three dimensional scene is mapped to a two dimensional image of the projection transformation is relatively complex.

\section{Principle of 3d Coordinate System.}

1) World Coordinate System. Usually the world coordinate system [1] is a three-dimensional Cartesian coordinate system. It is a global coordinate system, generally for the right-handed coordinate system. The entire graphics object in the coordinate system is mainly used for graphic scene space position, the position of the observer (perspective) and the definition of the line of sight, etc. Computer graphics system involved in the other coordinate system is basically referred to define it.

2) Local Coordinate System. For the convenience of geometric modeling and observation object, the world coordinate system defined two-dimensional or three-dimensional Cartesian coordinate system is called the local coordinate system. In local coordinate system defined in the "local" objects, by specifying a local coordinate system in the world coordinate system of the bearing, using geometry transform, it can be "local" defined object transform into the world coordinate system, make the upgrading of things in the world coordinate system.

3) Observe Coordinate System. Coordinate system is based on the position of the viewpoint for the origin, usually by the user to specify an up observation vector to define a coordinate system. Observation coordinate system is mainly used to from the Angle of the observer to look at the entire graphics object within the world coordinate system, in order to simplify the mathematics of geometric objects in visual plane become imaging plane.

3d Scene View Transform. The world coordinate system in the process of $3 \mathrm{~d}$ scene is mapped to a projection plane as perspective transformation [6]. Mathematical model for the matrix composite, fine a complete watch transformation needs the following properties:

1) Define view pointer, observing center and unit vector upward direction. The observation coordinate system and observation matrix will be decided.

2) Determine the projection plane. According to the observation center and the unit vector of observation direction, determine the projection plane based on space plane equation.

3) Determine the actual size of projection plane. Projection plane is as a rules of the rectangle, it is sized by plane's left boundary (left), right boundary (right) and the top boundary (top) and the bottom boundary (bottom). The length of projection plane is twice the left boundary (default left $=$ - right) and width is twice the top boundary (default top = - bottom).

4) Determine the front and back clip plane of the view-volume. In $3 \mathrm{~d}$ scene, after determining the position, direction and projection plane, observed model has been relative positioning, 
the observed model to evaluate relative to the point of view of recent cutting plane furthest distance $\mathrm{N}$ and cutting plane distance $\mathrm{F}$ that is to determine the minimum surrounded by observing body of the model.

5) Determine the projection matrix. Literature [9] provides a projection matrix method in determining the observed both the front and back clip plane, trying to attribute such as graphic size, need to pass the projection transformation will observe body is mapped to a standard observation in the body. Projection matrix is defined in the observation system, in observing the coordinate system is located in the origin, eyes to observe the direction for the $\mathrm{Z}$ axis direction, and to $\mathrm{Y}$ axis for the view of upward direction. Through the observation matrix transformation, the three dimensional objects vertex is transform to the space. Polygons may be for this time of the cone cutting, but by cutting in the irregular body is not so easy thing, so after graphics predecessors careful analysis, the cutting was arranged to standard observation in the body, the standard observation of body is a range of coordinates in $[-1,1]$ cube, polygon clipping is done with the rules of the body.

\section{Technical Solution}

According to the principle of the known technology, formulate the corresponding technical solution to achieve our requirements. First of all, according to the $3 \mathrm{~d}$ scene actual size to determine a minimum bounding box, and then according to the size of the three-dimensional space and location of the minimum bounding box to create scenes ball which is used to determine the observation matrix; According to the observation matrix to calculate the optimal projection matrix and determine the minimum observation, and view the results in the end.

Determine the minimum bounding box.

The minimum bounding box [3] is parallel to the minimum of the coordinate plane, each in three sets of hexahedron (rectangle) surrounded by a $3 \mathrm{~d}$ scene, refers to the hexahedron happens to be surrounded by the $3 \mathrm{~d}$ scene of the least of all the hexahedron. Minimum bounding box calculation method is as follows: a $3 \mathrm{~d}$ scene to all vertices $\mathrm{x}, \mathrm{y}$, and $\mathrm{z}$ coordinates, respectively, and the minimum and maximum $x, y$, and $z$ coordinates, named Xmin, Xmax, Ymin, Ymax, Zmin, Zmax, the smallest coordinates (Xmin, Ymin, Zmin) and maximum coordinates (Xmax, Ymax, Zmax) parallel to the plane coordinate of the rectangle is formed by the minimum bounding box.

Create a ball to observe the scene. For projection of three-dimensional objects, there is an infinite number of observation coordinate system to observe the changing. So the scene watch the ball created in the system, make its objects, surrounded by projection sphere radius size of stadia, set a certain number of points on a sphere, represent different viewpoints, point to the direction of its coordinates for the projection direction. In the second, literature $[10,11]$ based on point location of $3 \mathrm{~d}$ scene observation, there made improvements, sphere radius can be changed in the practical application, spherical coordinate points can pick up means point location, the projection direction can change, people can get different projection effect, greatly enhance the experimental efficiency and user interactivity.

1) The model of position location and the view direction.

a) Set the view center, the default for the parcel around the center of the box.

b) Calculate the minimum radius $\mathrm{R}$ of the view sphere that is the smallest line-of-sight (to ensure that $3 \mathrm{~d}$ scene is completely surrounded by the final view-volume).

c) As the core, to create view sphere with a radius $\mathrm{R}$ based the view center. The reference point on the spherical represent stadia $R$ position point of view, pointing to the center of the projection direction on behalf of the observation direction.

2) The mode of observe-to-upward direction. After determining the position location and direction there needs to set the up a vector $V_{u}=\left(x_{u}, y_{u}, z_{u}\right)$, we created visual model to select a reference point to set view-upward direction in the system.

Determine the minimum view-volume under the particular observation matrix body. After determining the position location, direction and observe upward direction, it needs to determine a 
minimum view-volume which can surround the $3 \mathrm{~d}$ scene according to the actual size of observed $3 \mathrm{~d}$ scene. In determining the minimum surrounded by observing body, need to compute the following attribute parameter:

1) Determine the projection plane size. In section 2 of the technical principle and determine the parameters in section 2 .

2) Determine the front, back clip plane distance. In determining the size of the projection plane, we need to further determine the watch body before and after the distance of the cutting plane, namely by each data point to determine all the plane parallel to the projection plane, find out the point of view to the plane of the minimum distance and maximum distance.

3) Validate parameters and display based on java3D [12].

\section{Experiment Result}

In the verification experiments, created as shown in figure 2, the three-dimensional mountain rendering model, figure 3 for grid said effect of the mountain.

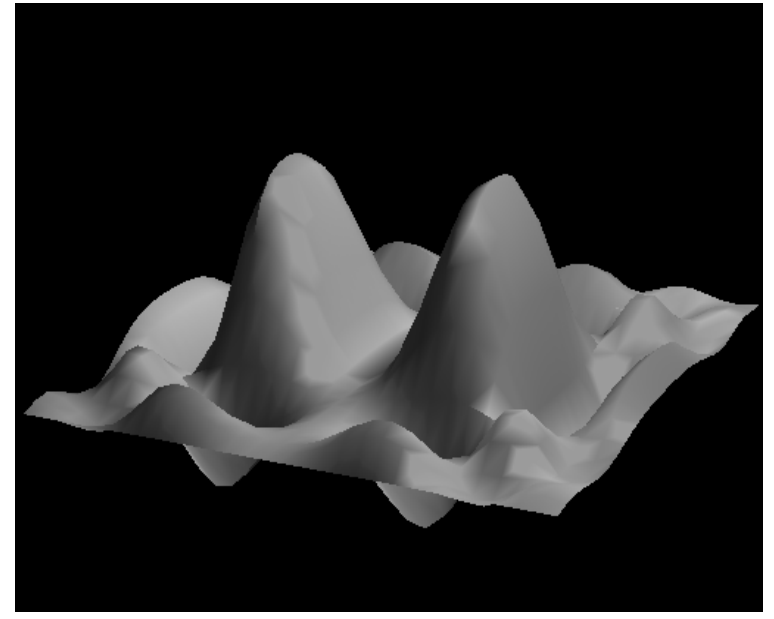

Figure $2.3 \mathrm{~d}$ mountain render model

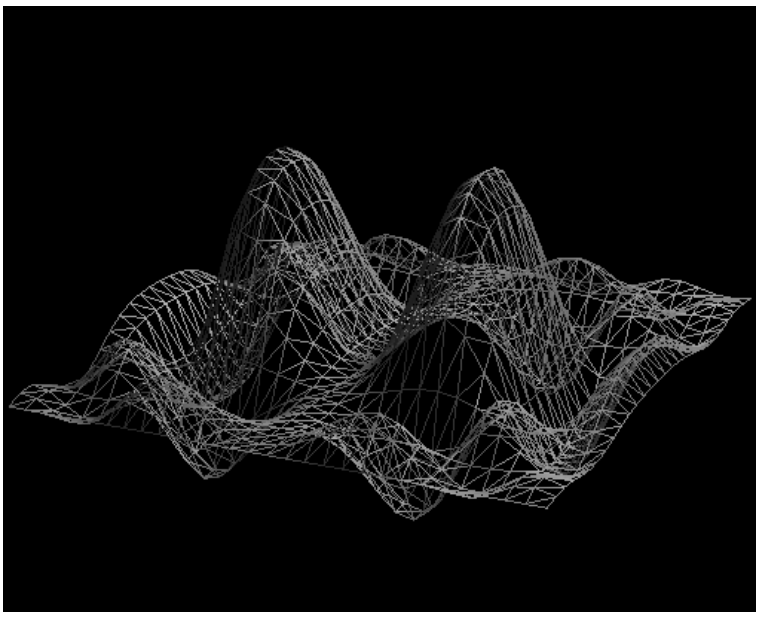

Figure 3.three-dimensional mountain grid model

As shown in figure 4, according to the size of the scene, the minimum bounding box and view center (the default is $3 \mathrm{~d}$ scene center) to calculate the minimum observation.

In testing system, selected two viewpoints on view sphere and observe the upward direction, generate the corresponding observation matrix. Experiment graph according to this method is shown as figure 5, corresponding to the observation matrix parameters shown in table 1; the view-volume parameters of experiment result is shown as figure 6, observation parameters shown in table 2.There made two contrast experiment 2 and experiment 3, the view-volume parameters is also shown in table 2. 


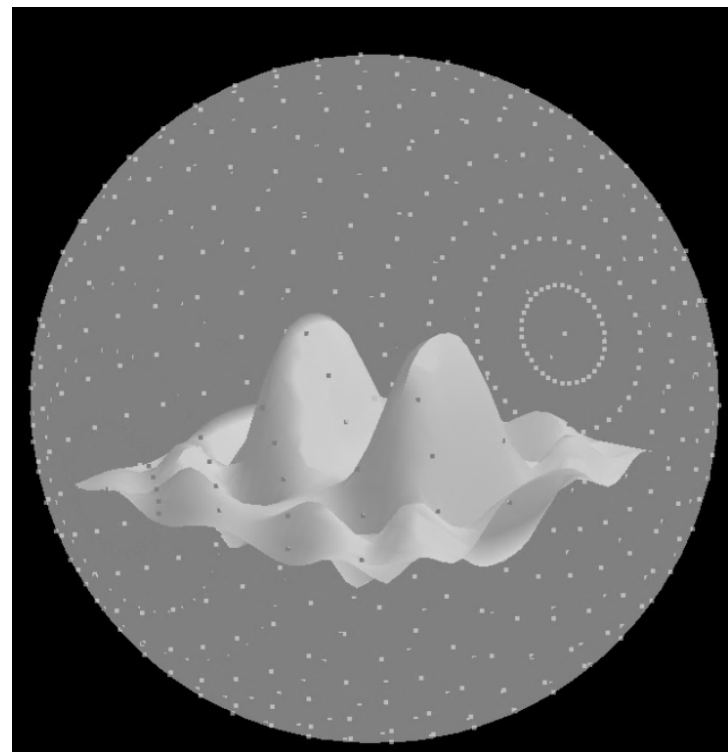

Figure 4.the smallest view sphere

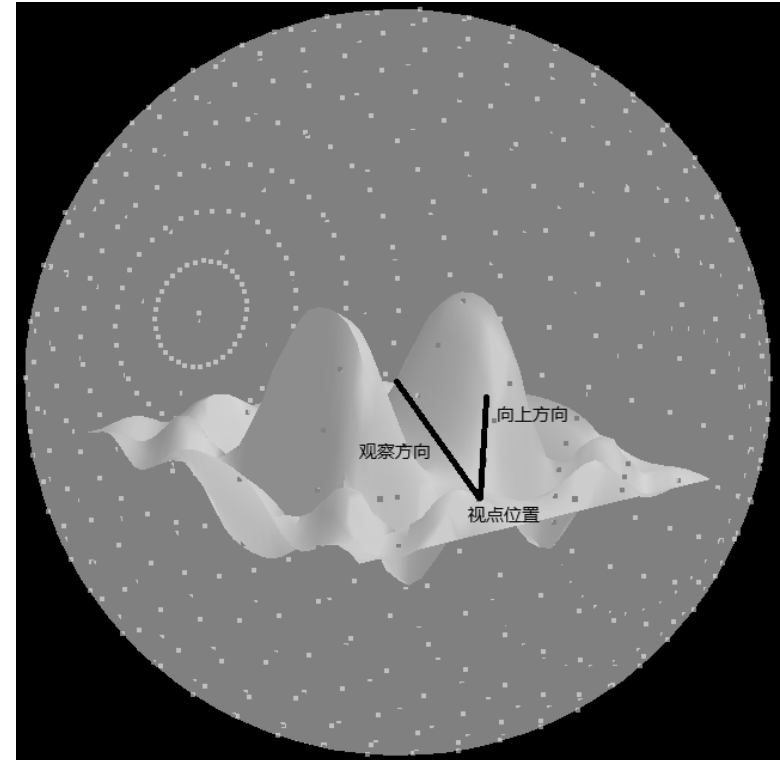

Figure 5.Experiment corresponding model of observation matrix

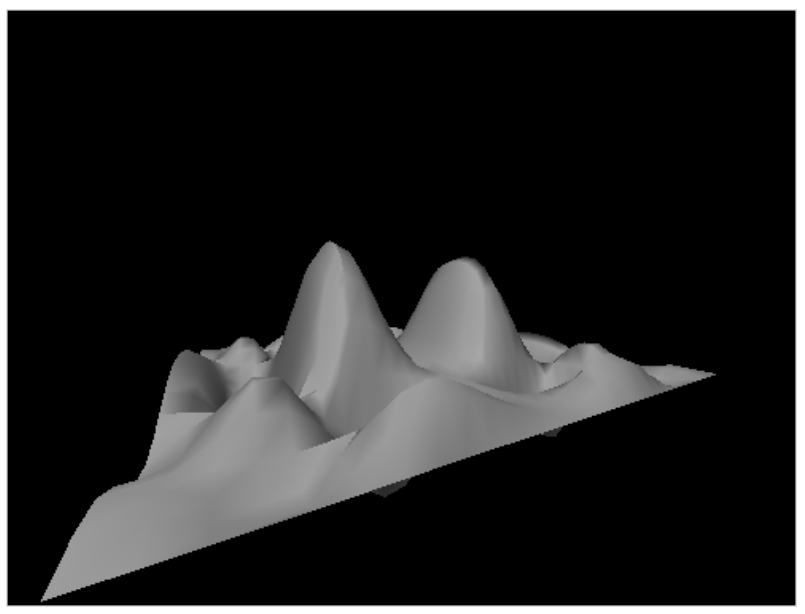

Figure 6.Experiment result

Table 1.Observation matrix test data

\begin{tabular}{|c|c|c|c|c|c|c|c|c|c|}
\hline & \multicolumn{3}{|c|}{ View center } & \multicolumn{3}{c|}{ View point } & \multicolumn{3}{c|}{ View-up direction } \\
\cline { 2 - 11 } & $\boldsymbol{X}$ & $\boldsymbol{Y}$ & $\boldsymbol{Z}$ & $\boldsymbol{X}$ & $\boldsymbol{Y}$ & $\boldsymbol{Z}$ & $\boldsymbol{X}$ & $\boldsymbol{Y}$ & $\boldsymbol{Z}$ \\
\hline Experiment & 0.00 & 0.00 & 0.00 & 4.04 & 0.00 & 2.33 & 0.00 & 1.00 & 0.00 \\
\hline
\end{tabular}

Table 2.The minimum view-volume parameter data

\begin{tabular}{|c|c|c|c|c|c|c|}
\hline & Left & Right & Bottom & Top & Near & far \\
\hline Experiment Result & -1.12 & 1.12 & -1.01 & 1.01 & 0.84 & 8.76 \\
\hline Experiment 2 & -1.05 & 1.05 & -1.01 & 1.01 & 0.84 & 8.76 \\
\hline Experiment 3 & -1.12 & 1.12 & -1.01 & 1.01 & 0.85 & 8.76 \\
\hline
\end{tabular}




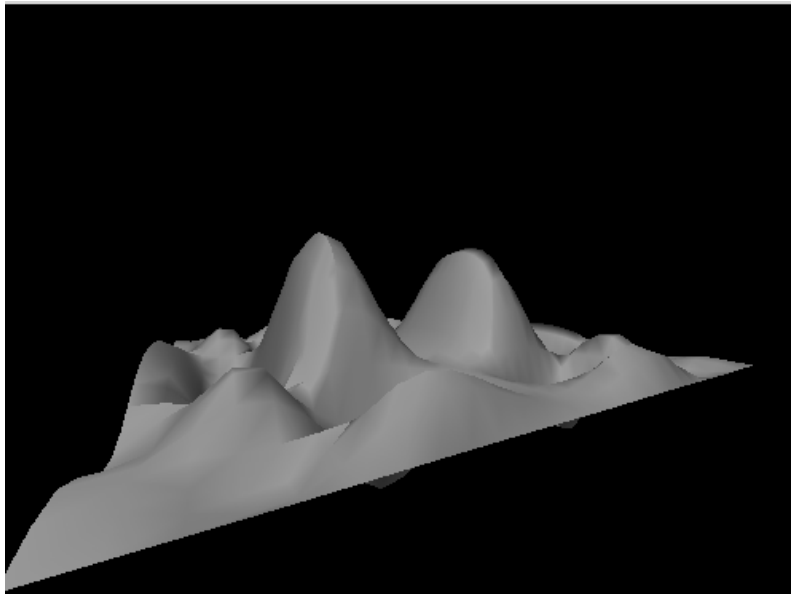

Figure 7.Result of Experiment 2

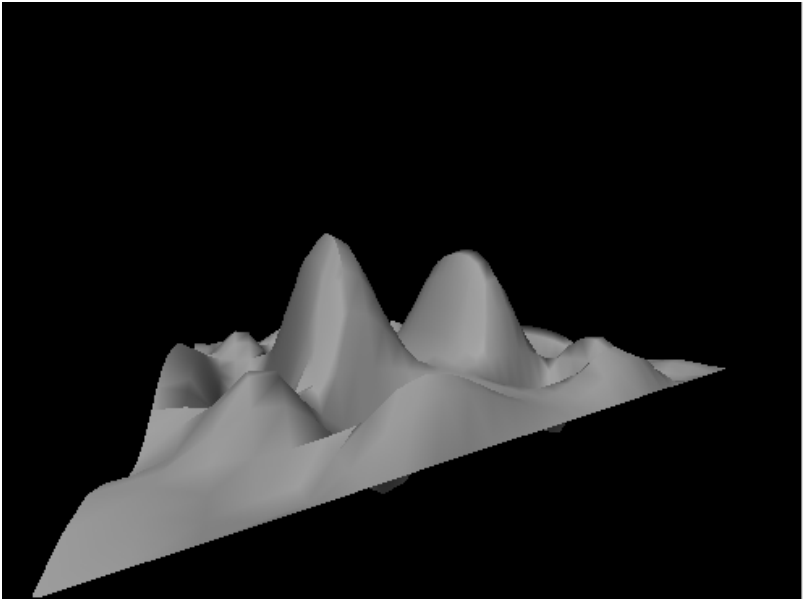

Figure 8.Result of Experiment 3

\section{Conclusion}

In view of some of the problems in the $3 \mathrm{~d}$ scene transform, such as $3 \mathrm{~d}$ scene and observe the cone changed, resulting in a defect view shows that cannot get ideal of projection views, brings to the system development work confusion and interference, etc., this article proposed the three-dimensional scene space volume and define a watch ball, point for point of view, the surface of a sphere projection plane at its center, determine the radius of the watch the ball makes the formation of the visual cone including $3 \mathrm{~d}$ scene; Then further to determine the optimal projection matrix (define minimum surrounded by observing body) won the improving method of the projection of the desired outcome can be effectively solved. In the future graphics system can be used, improve the system efficiency.

At the same time, some insufficiency in the method, such as view set reference point distribution is not uniform may lead to the point of view under the effect of projection is not easy to distinguish; Or data error leads to distortion of projection effect. These need to be improved in the future experiment research.

\section{Reference}

[1] Hong Zhang y. Daniel Liang. Computer graphics: using Java2D and 3D [M]. Beijing: mechanical industry press, 2008:218-222(In Chinese).

[2] J.J Li. The perspective transformation matrix of the initial parameters in the view and image position [J]. Journal of taiyuan university of science and technology, 1989(3):1-11(In Chinese).

[3] X.R Gao. Design and design of 3d dynamic graphics network in 3d and computer [M]. Beijing: tsinghua university press, 2013:231-232(In Chinese).

[4] Agrawala M. Zorin D. Munzner T. Artistic multi-projection rendering [C]: Euro-graphics Workshop on Rendering Techniques 2000, Brno, Czech Republic, June. 2000: 125-136.

[5] Singh K. A Fresh Perspective [J]. Graphics Interface, 2002, 46(4):129-130.

[6] Y.J Jiang. Realization of Three-Dimensional Viewing Transformation in World Scene [J]. Journal of Joshua University, 2006.

[7] D.R. Roberts, A.D. Marshall. A strategy for complete inspection of three-dimensional objects by active stereo computer vision. In International Workshop on Advanced Robotics and Intelligent Machines, April 1996.

[8] D.R. Roberts, A.D. Marshall. (1970). Viewpoint Selection for Complete Surface Coverage of Three Dimensional Objects [J]. Paper presented at the British Machine Vision Conference. 
[9] H Sun and Y zhang. The computation of projection matrix in computer vision camera. [J]. Journal of hebei normal university (natural science edition), 2002, 26(1): 26-28(In Chinese).

[10] Eades P, Houle M E, Webber R. Finding the best viewpoints for three-dimensional graph drawings [M]. Graph Drawing. Springer Berlin Heidelberg, 1970:87-98.

[11] Tarr M J, Williams P, Hayward W G, et al. Three-dimensional object recognition is viewpoint dependent [J]. Nature Neuroscience, 1998, 1(4):275-7.

[12] T Ji. Research based on 3d dynamic display in Java3D [J]. Journal of the tianjin vocational college, 2008, 10(5): 30-34(In Chinese). 